\title{
Impurity-induced double transitions for accidentally degenerate unconventional pairing states
}

\author{
Bastian Zinkl $\odot$ and Manfred Sigrist \\ Institute for Theoretical Physics, ETH Zurich, 8093 Zurich, Switzerland
}

(Received 26 September 2020; revised 6 December 2020; accepted 11 December 2020; published 14 January 2021)

\begin{abstract}
Nonmagnetic impurities can lift the accidental degeneracy of unconventional pairing states, such as the $(d+i g)$-wave state recently proposed for $\mathrm{Sr}_{2} \mathrm{RuO}_{4}$. This type of effect would lead to a superconducting double transition upon impurity doping. In a model calculation it is shown how this behavior depends on material parameters and how it could be detected.
\end{abstract}

DOI: 10.1103/PhysRevResearch.3.L012004

\section{INTRODUCTION}

The ideal proposal for the symmetry of the order parameter of an unconventional superconductor should have the ability to explain all its specific experimental signatures. In the case of $\mathrm{Sr}_{2} \mathrm{RuO}_{4}$, this high standard has turned out to be most challenging. Even the candidate order parameter considered as promising over a long time, the spin-triplet chiral $p$-wave state [1-4], has recently been questioned by contradictory experiments indicating spin-singlet pairing based on NMR Knight shift measurements [5-7]. Heat transport measurements and scanning tunneling microscopy quasiparticle interference experiments suggest the presence of line nodes, which was interpreted as evidence of $d$-wave pairing [8,9]. Experiments using uniaxial strain did not observe the splitting of the phase transition expected for the chiral $p$-wave state in the measurement of specific heat [10], while muon-spin rotation ( $\mu \mathrm{SR})$ results show the appearance of intrinsic magnetism indicating time-reversal symmetry breaking separate from the onset of superconductivity, consistent with chiral $p$-wave pairing [11].

These developments have prompted new proposals for the pairing symmetries, some of which have recently gained prominence, such as the even-parity, spin-singlet, timereversal symmetry breaking superposition of $d_{x^{2}-y^{2}}$ and $g_{x y\left(x^{2}-y^{2}\right)}$, the $(d+i g)$-wave state [12-14]. In contrast to the chiral $p$-wave state, whose two constituents, the $p_{x^{-}}$and $p_{y^{-}}$ components, are degenerate by symmetry, the $(d+i g)$-wave state has to rely on an accidental degeneracy, because $d_{x^{2}-y^{2}}$ and $g_{x y\left(x^{2}-y^{2}\right)}$ belong to different representations of the tetragonal point group. The choice of this combination was largely motivated by ultrasound measurements indicating a multicomponent order parameter in the superconducting phase, whereby the $(d+i g)$ combination is symmetrywise compatible. On the other hand, the analogous combination $(s+i d)$ wave would be in conflict with the ultrasound data [15].

Published by the American Physical Society under the terms of the Creative Commons Attribution 4.0 International license. Further distribution of this work must maintain attribution to the author(s) and the published article's title, journal citation, and DOI.
An alternative even-parity phase, which had been discussed in the past, is the chiral $d$-wave state, $d_{z x}+i d_{y z}$, whose two components are degenerate analogous to those of the chiral $p$ wave phase [13,16-18]. This state involves interlayer pairing and has a symmetry-imposed horizontal line node at $k_{z}=0$, which would fit well with the interpretation of the magnetic field angle dependence of the specific heat by Kittaka et al. [19]. This phase shares much of its phenomenology with the chiral $p$-wave state.

Here we examine specifically the $(d+i g)$-wave scenario regarding the aspect of degeneracy in view of disorder effects. While our study is motivated by $\mathrm{Sr}_{2} \mathrm{RuO}_{4}$, we do not attempt to give any quantitative discussion based on a realistic modeling of this multiband system. Rather we would like to demonstrate how disorder affects the proposed $(d+i g)$-wave state and what generally expected properties could be.

For this purpose, we formulated a single-band model and apply the self-consistent $T$-matrix approximation in order to take the effect of impurity scattering on the superconducting phase into account. In this way, we examine the behavior of the two pairing channels, in particular, the splitting of their transition temperatures.

\section{MODEL OF A $(d+i g)$-WAVE SUPERCONDUCTOR}

\section{A. Tight-binding model}

We consider a single-band tight-binding model on a twodimensional square lattice, which includes nearest-neighbor (NN) and next-nearest-neighbor (NNN) hopping. In momentum space the Hamiltonian reads

$$
\mathcal{H}=\sum_{\boldsymbol{k}, s} \xi_{\boldsymbol{k}} c_{\boldsymbol{k}, s}^{\dagger} c_{\boldsymbol{k}, s}+V_{\text {pair }},
$$

where $c_{k, s}^{\dagger}\left(c_{k, s}\right)$ denotes the creation (annihilation) operator of an electron with spin $s=\uparrow, \downarrow$ and momentum $\boldsymbol{k}=\left(k_{x}, k_{y}\right)$. The dispersion, which is chosen to qualitatively resemble the genuinely two-dimensional $\gamma$ band of $\mathrm{Sr}_{2} \mathrm{RuO}_{4}$ for a certain band filling, is given by

$$
\xi_{k}=-2 t\left(\cos k_{x}+\cos k_{y}\right)-4 t^{\prime} \cos k_{x} \cos k_{y}-\mu,
$$

with $\mu$ as the chemical potential and hopping matrix elements $t=1$ (unit of energy) and $t^{\prime}=0.3$ (the lattice constant $a$ is 


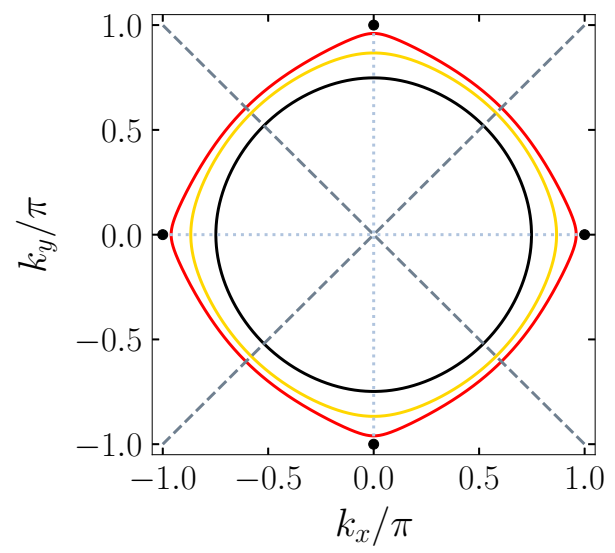

FIG. 1. Fermi surfaces for $\mu=0.25$ (black), $\mu=0.925$ (gold), and $\mu=1.175$ (red). The gap zeros of the $d$ wave are represented by the diagonal dashed lines (gray). The additional zeros of the $g$ wave are given by the horizontal and vertical dotted lines (light gray). The Van Hove points with a diverging density of states are marked by the black dots.

taken to unity). In Fig. 1 we show the Fermi surface (FS) for varying chemical potentials to demonstrate how such variations may influence the behavior of the superconducting phase qualitatively.

The pairing potential $V_{\text {pair }}$ is restricted to the spin-singlet channel,

$$
V_{\text {pair }}=\sum_{\substack{k, k^{\prime} \\ s_{1}, s_{2}}} V_{\boldsymbol{k} \boldsymbol{k}^{\prime}} c_{\boldsymbol{k}, s_{1}}^{\dagger} c_{-\boldsymbol{k},-s_{1}}^{\dagger} c_{-\boldsymbol{k}^{\prime},-s_{2}} c_{\boldsymbol{k}^{\prime}, s_{2}}
$$

where the orbital structure is given by $V_{k k^{\prime}}$. With our focus on the $(d+i g)$-wave [20], we introduce

$$
V_{\boldsymbol{k} \boldsymbol{k}^{\prime}}=\sum_{a=d, g} V_{a} \Phi_{a}(\boldsymbol{k}) \Phi_{a}\left(\boldsymbol{k}^{\prime}\right),
$$

where the even-parity basis functions are given by their lowest-order lattice harmonics,

$$
\begin{gathered}
\Phi_{d}(\boldsymbol{k})=\cos k_{x}-\cos k_{y}, \\
\Phi_{g}(\boldsymbol{k})=\sin k_{x} \sin k_{y}\left(\cos k_{x}-\cos k_{y}\right) .
\end{gathered}
$$

Within the square lattice approach these correspond to realspace pairing interactions between the closest sites capable of resolving the corresponding symmetry, i.e., between nearestneighbor (next-to-next-nearest-neighbor) sites for the $d$-wave ( $g$-wave) component. After the standard mean-field decoupling of the pairing potential, the minimization of the free energy leads naturally to the quasiparticle gap function

$$
\begin{aligned}
\Delta_{k}= & \Delta_{d}\left(\cos k_{x}-\cos k_{y}\right) \\
& \pm i \Delta_{g} \sin k_{x} \sin k_{y}\left(\cos k_{x}-\cos k_{y}\right),
\end{aligned}
$$

which breaks time-reversal symmetry. The coefficients $\Delta_{d, g}$ are obtained by solving the self-consistency equation,

$$
\left(\begin{array}{c}
\Delta_{d} \\
\Delta_{g}
\end{array}\right)=\sum_{k} \mathcal{C}_{\boldsymbol{k}}\left(\begin{array}{cc}
V_{d} & 0 \\
0 & V_{g} \sin ^{2} k_{x} \sin ^{2} k_{y}
\end{array}\right)\left(\begin{array}{c}
\Delta_{d} \\
\Delta_{g}
\end{array}\right) .
$$

The factor $C_{k}$ takes the form

$$
\mathcal{C}_{k}=-T \sum_{n} \frac{\left(\cos k_{x}-\cos k_{y}\right)^{2}}{\tilde{\omega}_{n}^{2}+\xi_{k}^{2}+\left|\Delta_{k}\right|^{2}},
$$

where $T$ is the temperature and the renormalized Matsubara frequencies $\tilde{\omega}_{n}$ are different from the standard fermionic ones, $\omega_{n}=(2 n+1) \pi k_{B} T$, if disorder is present, as defined in Eq. (13).

\section{B. Disorder- $T$-matrix approximation}

We introduce disorder through nonmagnetic impurities with a pointlike potential leading exclusively to $s$-wave scattering. As we would like to explore the whole range of scattering potential strengths, meaning also the unitary limit where the potential exceeds the bandwidth, we employ a $T$ matrix approach, which includes multiple scatterings at the same impurity. The $T$ matrix is defined by

$$
T_{\boldsymbol{k} \boldsymbol{k}^{\prime}}\left(i \omega_{n}\right)=U_{\boldsymbol{k} \boldsymbol{k}^{\prime}}+\sum_{\boldsymbol{k}^{\prime \prime}} U_{\boldsymbol{k} \boldsymbol{k}^{\prime \prime}} G\left(\boldsymbol{k}^{\prime \prime}, i \omega_{n}\right) T_{\boldsymbol{k}^{\prime \prime} \boldsymbol{k}^{\prime}}\left(i \omega_{n}\right),
$$

where $U_{\boldsymbol{k} \boldsymbol{k}^{\prime}}$ is the impurity potential in $\boldsymbol{k}$ space and $G\left(\boldsymbol{k}, i \omega_{n}\right)$ the (normal) electron Green's function. Note that we have omitted off-diagonal terms involving the anomalous Green's function, since they vanish for unconventional states. For $s$-wave scattering both $U_{k k^{\prime}}$ and the $T$ matrix are scalar in momentum space,

$$
U_{\boldsymbol{k} \boldsymbol{k}^{\prime}}=U, \quad T_{\boldsymbol{k} \boldsymbol{k}^{\prime}}\left(i \omega_{n}\right)=T\left(i \omega_{n}\right) .
$$

We may restrict ourselves to low impurity concentrations $c$ such that we can neglect impurity interference effects, because superconductivity is rather quickly suppressed by disorder, once the mean free path becomes comparable to the zerotemperature coherence length. Hence, the self-energy reads

$$
\Sigma\left(i \omega_{n}\right)=c T\left(i \omega_{n}\right),
$$

which renormalizes the Matsubara frequencies,

$$
i \tilde{\omega}_{n}=i \omega_{n}-\Sigma\left(i \omega_{n}\right) .
$$

Using the renormalized frequencies $\tilde{\omega}_{n}$ in the self-consistent gap equation [Eqs. (8) and (9)] enables us to examine the influence of disorder on the superposition of unconventional pairing states.

\section{Critical temperatures $\boldsymbol{T}_{c, d}$ and $\boldsymbol{T}_{c, g}$}

For two pairing states, which belong to different representations, such as the $d$ - and $g$-wave states, the respective bare critical temperatures, $T_{c, d}$ and $T_{c, g}$, are generally different. In Appendix A we discuss briefly the related case of the $(s+i d)$ wave.

We now assume that the critical temperatures coincide in the clean system and enforce this in our model by finetuning the coupling strengths $V_{d, g}$ in the pairing interaction accordingly. Focusing on the behavior of the bare critical temperatures, $T_{c, d}$ and $T_{c, g}$, under the influence of disorder, we solve the linearized gap equation [Eq. (8)], which decouples for the two channels. The ratio $T_{c, d} / T_{c, g}$ displayed in Fig. 2 (circles) reveals two regimes, if we vary the chemical potential. For $\mu=0.25$ (smallest FS) the ratio $T_{c, d} / T_{c, g}$ decreases 


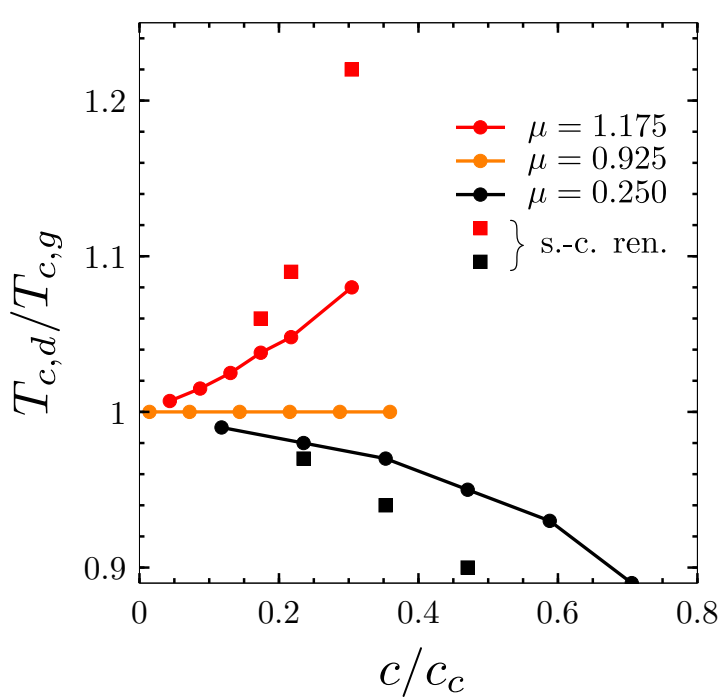

FIG. 2. The ratio of the critical temperatures, $T_{c, d} / T_{c, g}$, as a function of the impurity concentration for different values of the chemical potential, $\mu$. We normalize the concentration values by $c_{c}$, which is the average of the critical concentrations, $\left(c_{c, d}+c_{c, g}\right) / 2$. The bare (renormalized) critical temperatures obtained from the linearized (full) self-consistent gap equations are given by the dots (squares).

upon growing impurity concentration $c$, while it increases for $\mu=1.175$ (largest FS close to Van Hove points). No change of the ratio is seen for $\mu=0.925$. Thus, there is a fine-tuned FS where the "degeneracy" remains essentially untouched.

The difference in the behavior is reflected in the coherence lengths of the two pairing states, which depend on the position of the FS. A simple estimate of the zero-temperature coherence length $\xi$ for a given gap function can be obtained from

$$
\xi^{2}=\frac{\sum_{k}\left|\nabla_{k} \frac{\Delta_{k}}{E_{k}}\right|^{2}}{\sum_{k}\left|\frac{\Delta_{k}}{E_{k}}\right|^{2}} .
$$

For larger coherence lengths $T_{c}$ suffers faster suppression with increasing $c$. Consistently, we find $\xi_{d} / \xi_{g} \approx 1.09$ for $\mu=0.25$ and $\xi_{d} / \xi_{g} \approx 0.96$ for $\mu=1.175$. Intuitively it is clear for the latter case that the $d$-wave state can profit from the larger density of states at the Van Hove points (small Fermi velocity), while the $g$-wave state has nodes there. Hence, the $d$-wave state incorporates more tightly bound Cooper pairs. However, on more genuine Fermi surfaces pairing states of higher angular momentum have in general shorter coherence lengths for a given critical temperature.

The splitting of the bare critical temperatures implies the occurrence of two consecutive superconducting transitions: First, into the superconducting phase and then breaking timereversal symmetry. The second transition, however, does not happen at the lower of the two bare $T_{c}$, but at a renormalized critical temperature, because the second-order parameter has to nucleate in the presence of the first one. Thus, to determine the real onset of the second-order parameter we have to solve the full self-consistency equation [Eq. (8)] for $\Delta_{d}$ and $\Delta_{g}$. The renormalization of the critical temperatures, indicated by squares in Fig. 2, yields a larger splitting of the two transitions than the ratio $T_{c, d} / T_{c, g}$ would suggest. Due to the presence of the first-order parameter large parts of the states at the FS are

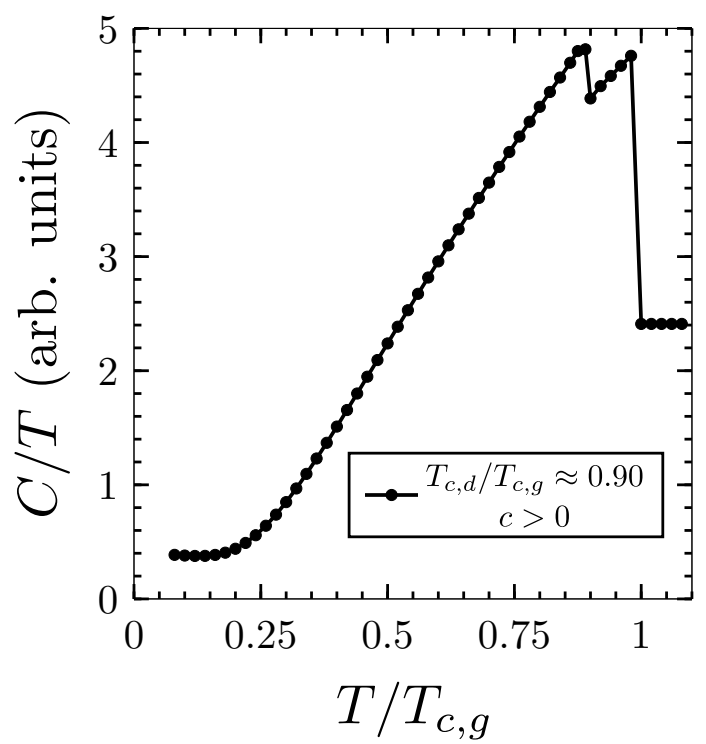

FIG. 3. The specific heat divided through temperature, $C / T$, as a function of temperature $T$ for a finite impurity concentration, $c>0$. The ratio of the critical temperatures is given by $T_{c, d} / T_{c, g} \approx 0.90$ and the chemical potential by $\mu=0.25$.

consumed leaving a strongly reduced density of low-energy states available for the second-order parameter.

\section{Detecting the double transition}

There are few ways of observing superconducting double transitions. Traditionally, specific heat has been a hallmark of such a feature in many unconventional superconductors. Thus, we would like to show here that the impurity-induced split of the transition could leave an observable signature in the specific heat. We use our Green's function formalism and linear response theory [21,22], as shown in Appendix B.

We consider here the situation $T_{c, g}>T_{c, d}$, where the first transition leads to a $g$-wave phase and the second to the time-reversal symmetry breaking $(d+i g)$-wave phase. Figure 3 depicts the temperature dependence of the specific heat, $C / T$. Clearly a second anomaly is visible below the onset of superconductivity. For the parameter chosen in our calculation the second jump is well visible with a size of roughly $20 \%$ of the first one and both transitions are of second order. The jump size can, however, depend on details of the electronic structure and the pairing states. Note that $C / T$ reaches a finite value in the zero-temperature limit due to the finite zero-energy density of states induced by the disorder.

An alternative way to detect the double transition is the measurement of ultrasound velocity, which would show two consecutive steps of renormalization, as for example seen in $\mathrm{UPt}_{3}$ [23]. An important feature is the violation of timereversal symmetry at the second transition introducing the intrinsic magnetism so far observed at the onset temperature. This means that the separation of the increase of the $\mu \mathrm{SR}$ zero-field relaxation rate from the onset of superconductivity would be a decisive feature, as known from $\mathrm{UTh}_{x} \mathrm{Be}_{13-x}$ [24]. Alternatively, the polar Kerr effect has been recently used to observe the violation of time-reversal symmetry at the second transition of $\mathrm{UPt}_{3}$ [25]. 


\section{CONCLUSION}

Our work highlights how nonmagnetic disorder influences the transition temperatures of accidentally or nearly degenerate unconventional pairing channels. Generally, the two pairing states would show a different suppression of their critical temperatures under disorder, which would cause a superconducting double transition. Such a double transition would be visible as a double jump in the specific heat, as shown in Fig. 3, or as two anomalies in the ultrasound velocity. However, since time-reversal symmetry breaking would only occur at the second transition, $\mu \mathrm{SR}$ zero-field relaxation and polar Kerr effect measurements would be optimal tools to detect whether the appearance of intrinsic magnetic properties separates from the onset of superconductivity. So far no such features have been reported despite the fact that many samples with rather different critical temperatures have been investigated over time, and, therefore, should be indeed a target of more systematic measurements. The scenario based on the $(d+i g)$-wave phase for $\mathrm{Sr}_{2} \mathrm{RuO}_{4}$ relies on fine-tuning in the clean limit. We show that additional fine-tuning could, in principle, conserve the accidental degeneracy even under disorder. Nevertheless, a second means to eliminate such degeneracy is provided by hydrostatic pressure, since for order parameters, which are accidentally degenerate, the pressure dependence of their $T_{c}$ would be generally different, i.e., $d T_{c, d} / d p \neq d T_{c, g} / d p$. This difference is not connected to the dependence of $T_{c}$ on disorder such that a further accidental fine-tuning would be rather unlikely. Finally, we would like to emphasize that the absence of a splitting of the transition under both disorder and pressure would be compatible with both the chiral $p$-wave $\left(p_{x}+i p_{y}\right)$ and the chiral $d$-wave $\left(d_{z x}+i d_{y z}\right)$ states, whereby the latter has the advantage of being straightforwardly consistent with the Knight shift data.

\section{ACKNOWLEDGMENTS}

We would like to thank Mark H. Fischer, Roland Willa, Brad Ramshaw, Roustem Khassanov, Das Debarchan, Ritu Gupta, and Vadim Grinenko for insightful discussions. This work was financially supported by the Swiss National Science Foundation (SNSF) through Division II (Grant No. 184739).

\section{APPENDIX A: DISORDER EFFECT ON THE $(s+i d)$-WAVE PHASE}

For completeness we address here also an alternative state proposed, which constitutes the superposition of the extended $s$-wave and $d$-wave states which would not be degenerate by symmetry. The gap equations read

$$
\left(\begin{array}{c}
\Delta_{s} \\
\Delta_{d}
\end{array}\right)=\sum_{\boldsymbol{k}} \mathcal{C}_{\boldsymbol{k}}^{\prime}\left(\begin{array}{cc}
V_{s} \Phi_{s}(\boldsymbol{k})^{2} & 0 \\
0 & V_{d} \Phi_{d}(\boldsymbol{k})^{2}
\end{array}\right)\left(\begin{array}{c}
\Delta_{s} \\
\Delta_{d}
\end{array}\right)
$$

with

$$
\begin{aligned}
& \Phi_{s}(\boldsymbol{k})=\cos k_{x}+\cos k_{y}, \\
& \Phi_{d}(\boldsymbol{k})=\cos k_{x}-\cos k_{y},
\end{aligned}
$$

TABLE I. The ratio of critical temperatures of the $(s+i d)$ wave at $\mu=1.175$ as a function of the impurity concentration $c$. The average of the critical concentrations, $c_{c, s} \approx 0.057$ and $c_{c, d} \approx 0.128$, is denoted by $c_{c}$.

\begin{tabular}{cc}
\hline \hline$c / c_{c}$ & $T_{c, s} / T_{c, d}$ \\
\hline 0.11 & 0.887 \\
0.16 & 0.824 \\
0.22 & 0.755 \\
\hline \hline
\end{tabular}

and

$$
\mathcal{C}_{k}^{\prime}=-T \sum_{n} \frac{1}{\tilde{\omega}_{n}^{2}+\xi_{k}^{2}+\left|\Delta_{k}\right|^{2}} .
$$

As explained in the main text, we calculate the bare critical temperatures, $T_{c, s}$ and $T_{c, d}$, from the decoupled linearized gap equations of the two pairing channels. As a representative case we chose $\mu=1.175$ (largest FS in Fig. 1) and list the results for different impurity concentrations in Table I. The impurity concentration is normalized by the averaged critical concentration $c_{c}=\left(c_{s}+c_{d}\right) / 2$. Assuming degeneracy in the clean system, we find that the ratio $T_{c, s} / T_{c, d}$ is decreasing as a function of the impurity concentration, which is in line with the ratio of coherence lengths, $\xi_{s} / \xi_{d} \approx 1.36$. We checked that the qualitative behavior of $T_{c, s} / T_{c, d}$ to decrease under impurity doping is independent of $\mu$, in contrast to the $(d+i g)$ pairing state (cf. Fig. 2).

\section{APPENDIX B: CALCULATION OF THE SPECIFIC HEAT IN DISORDERED SYSTEMS}

For the derivation of the specific heat we employ the Green's function formalism and linear response theory $[26,27]$. We start with the generalized formula for the groundstate energy of an interacting electron system by Luttinger and Ward $[21,22]$. The grand potential can be written as

$$
\begin{gathered}
\Omega_{s}=-T \sum_{n} \sum_{\boldsymbol{k}}\left\{\log \left(\tilde{\omega}_{n}^{2}+\xi_{\boldsymbol{k}}^{2}+\left|\Delta_{\boldsymbol{k}}\right|^{2}\right)+\Delta_{\boldsymbol{k}} F^{\dagger}\left(\boldsymbol{k}, i \omega_{n}\right)\right. \\
\left.+\Sigma\left(i \omega_{n}\right) G\left(\boldsymbol{k}, i \omega_{n}\right)\right\}+\Omega^{\prime}, \\
\text { with } i \tilde{\omega}_{n}=i \omega_{n}-\Sigma\left(i \omega_{n}\right) \text { and } \\
\Omega^{\prime}=T \sum_{v} \sum_{n} \sum_{\boldsymbol{k}} \frac{1}{v} \Sigma_{v}\left(i \omega_{n}\right) G\left(\boldsymbol{k}, i \omega_{n}\right),
\end{gathered}
$$

where $\Sigma\left(i \omega_{n}\right)=c T\left(i \omega_{n}\right)=\sum_{v} \Sigma_{v}\left(i \omega_{n}\right)$. The normal and anomalous Green's functions are given by

$$
\begin{gathered}
G\left(\boldsymbol{k}, i \omega_{n}\right)=-\frac{i \tilde{\omega}_{n}+\xi_{\boldsymbol{k}}}{\tilde{\omega}_{n}^{2}+\xi_{\boldsymbol{k}}^{2}+\left|\Delta_{\boldsymbol{k}}\right|^{2}}, \\
F^{\dagger}\left(\boldsymbol{k}, i \omega_{n}\right)=\frac{\Delta_{\boldsymbol{k}}^{*}}{\tilde{\omega}_{n}^{2}+\xi_{\boldsymbol{k}}^{2}+\left|\Delta_{\boldsymbol{k}}\right|^{2}} .
\end{gathered}
$$

By considering the difference between superconducting and normal state, $\Omega_{s}-\Omega_{n}$, we ensure that the sum over $n$ converges. After calculating the self-energy self-consistently it is straightforward to determine the specific heat difference 
through

$$
\frac{C_{s}-C_{n}}{T}=-\frac{\partial^{2}\left(\Omega_{s}-\Omega_{n}\right)}{\partial T^{2}} .
$$

The derivatives for the displayed results in Fig. 3 have been taken numerically.
[1] G. M. Luke, Y. Fudamoto, K. Kojima, M. Larkin, J. Merrin, B. Nachumi, Y. Uemura, Y. Maeno, Z. Mao, Y. Mori et al., Timereversal symmetry-breaking superconductivity in $\mathrm{Sr}_{2} \mathrm{RuO}_{4}$, Nature (London) 394, 558 (1998).

[2] A. P. Mackenzie and Y. Maeno, The superconductivity of $\mathrm{Sr}_{2} \mathrm{RuO}_{4}$ and the physics of spin-triplet pairing, Rev. Mod. Phys. 75, 657 (2003).

[3] J. Xia, Y. Maeno, P. T. Beyersdorf, M. M. Fejer, and A. Kapitulnik, High Resolution Polar Kerr Effect Measurements of $\mathrm{Sr}_{2} \mathrm{RuO}_{4}$ : Evidence for Broken Time-Reversal Symmetry in the Superconducting State, Phys. Rev. Lett. 97, 167002 (2006).

[4] Y. Maeno, S. Kittaka, T. Nomura, S. Yonezawa, and K. Ishida, Evaluation of spin-triplet superconductivity in $\mathrm{Sr}_{2} \mathrm{RuO}_{4}, \mathrm{~J}$. Phys. Soc. Jpn. 81, 011009 (2011).

[5] A. Pustogow, Y. Luo, A. Chronister, Y.-S. Su, D. Sokolov, F. Jerzembeck, A. P. Mackenzie, C. Hicks, N. Kikugawa, S. Raghu et al., Constraints on the superconducting order parameter in $\mathrm{Sr}_{2} \mathrm{RuO}_{4}$ from oxygen-17 nuclear magnetic resonance, Nature (London) 574, 72 (2019).

[6] K. Ishida, M. Manago, K. Kinjo, and Y. Maeno, Reduction of the ${ }^{17} \mathrm{O}$ knight shift in the superconducting state and the heatup effect by NMR pulses on $\mathrm{Sr}_{2} \mathrm{RuO}_{4}$, J. Phys. Soc. Jpn. 89, 034712 (2020).

[7] A. Chronister, A. Pustogow, N. Kikugawa, D. A. Sokolov, F. Jerzembeck, C. W. Hicks, A. P. Mackenzie, E. D. Bauer, and S. E. Brown, Evidence for even parity unconventional superconductivity in $\mathrm{Sr}_{2} \mathrm{RuO}_{4}$, arXiv:2007.13730.

[8] E. Hassinger, P. Bourgeois-Hope, H. Taniguchi, S. Rene de Cotret, G. Grissonnanche, M. S. Anwar, Y. Maeno, N. Doiron-Leyraud, and L. Taillefer, Vertical Line Nodes in the Superconducting Gap Structure of $\mathrm{Sr}_{2} \mathrm{RuO}_{4}$, Phys. Rev. X 7, 011032 (2017).

[9] R. Sharma, S. D. Edkins, Z. Wang, A. Kostin, C. Sow, Y. Maeno, A. P. Mackenzie, J. C. S. Davis, and V. Madhavan, Momentum-resolved superconducting energy gaps of $\mathrm{Sr}_{2} \mathrm{RuO}_{4}$ from quasiparticle interference imaging, Proc. Natl. Acad. Sci. U. S. A. 117, 5222 (2020).

[10] Y. S. Li, N. Kikugawa, D. A. Sokolov, F. Jerzembeck, A. S. Gibbs, Y. Maeno, C. W. Hicks, M. Nicklas, and A. P. Mackenzie, High sensitivity heat capacity measurements on $\mathrm{Sr}_{2} \mathrm{RuO}_{4}$ under uniaxial pressure, arXiv:1906.07597.

[11] V. Grinenko, S. Ghosh, R. Sarkar, J.-C. Orain, A. Nikitin, M. Elender, D. Das, Z. Guguchia, F. Brückner, M. E. Barber et al., Split superconducting and time-reversal symmetry-breaking transitions, and magnetic order in $\mathrm{Sr}_{2} \mathrm{RuO}_{4}$ under uniaxial stress, arXiv:2001.08152.

[12] S. A. Kivelson, A. C. Yuan, B. Ramshaw, and R. Thomale, A proposal for reconciling diverse experiments on the superconducting state in $\mathrm{Sr}_{2} \mathrm{RuO}_{4}$, npj Quantum Mater. 5, 43 (2020).
[13] S. Ghosh, A. Shekhter, F. Jerzembeck, N. Kikugawa, D. A. Sokolov, M. Brando, A. P. Mackenzie, C. W. Hicks, and B. J. Ramshaw, Thermodynamic evidence for a two-component superconducting order parameter in $\mathrm{Sr}_{2} \mathrm{RuO}_{4}$, Nat. Phys. (2020), doi:10.1038/s41567-020-1032-4.

[14] R. Willa, Symmetry-mixed bound-state order, Phys. Rev. B 102, 180503(R) (2020).

[15] A. T. Rømer, D. D. Scherer, I. M. Eremin, P. J. Hirschfeld, and B. M. Andersen, Knight Shift and Leading Superconducting Instability from Spin Fluctuations in $\mathrm{Sr}_{2} \mathrm{RuO}_{4}$, Phys. Rev. Lett. 123, 247001 (2019).

[16] I. Žutić and I. Mazin, Phase-Sensitive Tests of the Pairing State Symmetry in $\mathrm{Sr}_{2} \mathrm{RuO}_{4}$, Phys. Rev. Lett. 95, 217004 (2005).

[17] H. G. Suh, H. Menke, P. M. R. Brydon, C. Timm, A. Ramires, and D. F. Agterberg, Stabilizing even-parity chiral superconductivity in $\mathrm{Sr}_{2} \mathrm{RuO}_{4}$, Phys. Rev. Res. 2, 032023 (2020).

[18] H. S. Røising, T. Scaffidi, F. Flicker, G. F. Lange, and S. H. Simon, Superconducting order of $\mathrm{Sr}_{2} \mathrm{RuO}_{4}$ from a threedimensional microscopic model, Phys. Rev. Res. 1, 033108 (2019).

[19] S. Kittaka, S. Nakamura, T. Sakakibara, N. Kikugawa, T. Terashima, S. Uji, D. A. Sokolov, A. P. Mackenzie, K. Irie, Y. Tsutsumi et al., Searching for gap zeros in $\mathrm{Sr}_{2} \mathrm{RuO}_{4}$ via field-angle-dependent specific-heat measurement, J. Phys. Soc. Jpn. 87, 093703 (2018).

[20] Another even-parity state is, for instance, the superposition of extended $s$ wave and $d$ wave, for which we simply use the basis function $\Phi_{s}(\boldsymbol{k})=\cos k_{x}+\cos k_{y}$.

[21] J. M. Luttinger and J. C. Ward, Ground-state energy of a manyfermion system. II, Phys. Rev. 118, 1417 (1960).

[22] J. Keller, K. Scharnberg, and H. Monien, Free energy of anisotropic superconductors, Physica C 152, 302 (1988).

[23] G. Bruls, D. Weber, B. Wolf, P. Thalmeier, B. Lüthi, A. de Visser, and A. Menovsky, Strain-Order-Parameter Coupling and Phase Diagrams in Superconducting $\mathrm{UPt}_{3}$, Phys. Rev. Lett. 65, 2294 (1990).

[24] R. H. Heffner, J. O. Willis, J. L. Smith, P. Birrer, C. Baines, F. N. Gygax, B. Hitti, E. Lippelt, H. R. Ott, A. Schenck, and D. E. MacLaughlin, Muon-spin relaxation studies of weak magnetic correlations in $\mathrm{U}_{1-\mathrm{x}} \mathrm{Th}_{\mathrm{x}} \mathrm{Be}_{13}$, Phys. Rev. B 40, 806 (1989).

[25] E. R. Schemm, W. J. Gannon, C. M. Wishne, W. P. Halperin, and A. Kapitulnik, Observation of broken time-reversal symmetry in the heavy-fermion superconductor $\mathrm{UPt}_{3}$, Science 345, 190 (2014).

[26] V. P. Mineev and K. Samokhin, Introduction to Unconventional Superconductivity (Gordon and Breach, New York, 1999).

[27] T. Nomura, Theory of transport properties in the $p$-wave superconducting state of $\mathrm{Sr}_{2} \mathrm{RuO}_{4}-\mathrm{A}$ microscopic determination of the gap structure, J. Phys. Soc. Jpn. 74, 1818 (2005). 